\title{
A low PAPR performance with new segmentation schemes of partial transmit sequence for OFDM systems
}

\author{
Yasir A. Jawhar ${ }^{1, *}$, Nor Shahida Shah ${ }^{1}$, Montadar A. Taher ${ }^{2}$, Khairun N. Ramli ${ }^{1}$, Mustafa S. Ahmed ${ }^{1}$, R. \\ Abdulhasan ${ }^{1}$ \\ ${ }^{1}$ Faculty of Electrical and Electronic Engineering, Universiti Tun Hussein Onn Malaysia, Parit Raja, Batu Pahat, Johor, Malaysia \\ 2Department of Communications Engineering, College of Engineering, University of Diyala, Ba'aqubah, Diyala, Iraq
}

\section{ART ICLE INFO}

Article history:

Received 19 December 2016

Received in revised form

8 February 2017

Accepted 9 February 2017

Keywords:

PTS

OFDM

IL-PTS

Ad-PTS

Computational complexity

\begin{abstract}
A B S T R A C T
Despite the fact that OFDM has numerous features, the high peak-to-average power ratio (PAPR) deems one of the challenging disadvantages face by the system in the real applications. The high PAPR limits the OFDM signal efficiency on the transmitter side. Thus many PAPR alleviation approaches have been suggested in the past. Partial transmit sequences (PTS) has been accounted as one of the successful procedures for decreasing the high PAPR; with the consideration that the computational complexity of the PTS method regarded high relatively. Moreover, improving the PAPR mitigation performance of the PTS algorithm relies upon the number of the partitioned subblocks, the number of the phase rotation vectors, and the kind of the segmentation scheme utilized. This article presents new segmentation schemes to enhance the PAPR mitigation execution without further intricacy on the system. Adjacent shifting segmentation scheme (Ad-Sh-PTS) and rows exchange of interleaving segmentation schemes (IL-Ex-PTS) are proposed depending on the two well-known segmentation schemes of adjacent partition PTS (Ad-PTS) and interleaving partition PTS (IL-PTS). The simulation demonstrates that the suggested algorithms can accomplish mitigation in PAPR preferable compared to the traditional schemes, Ad-PTS and IL-PTS schemes.
\end{abstract}

(C) 2017 The Authors. Published by IASE. This is an open access article under the CC BY-NC-ND license (http://creativecommons.org/licenses/by-nc-nd/4.0/).

\section{Introduction}

Nowadays, the demand of the high-speed data rate applications led to developing the wireless communication frameworks rapidly. Through the last two decades, the wireless communication networks have been expanded significantly from the first generation (1G) to achieve the fourth generation (4G) these days. The development of the high-speed wireless communication systems was required by employing modulation schemes and data transmission techniques more advanced. OFDM is a modern multi-carrier method that broadly utilized as a part of high data rate of wireless frameworks, this due to its features over other techniques such as, its capacity for high information rate transmission, strength against channel fading, utilization the bandwidth efficiently, and high productivity (Rahmatallah and Mohan, 2013;

\section{* Corresponding Author.}

Email Address: free_cccc@yahoo.com (Y. A. Jawhar) https://doi.org/10.21833/ijaas.2017.04.003

2313-626X/C) 2017 The Authors. Published by IASE. This is an open access article under the CC BY-NC-ND license (http://creativecommons.org/licenses/by-nc-nd/4.0/)
Taspinar and Bozkurt, 2016; Praveenkumar et al., 2013; Vidya et al., 2015).

Moreover, numerous wireless frameworks utilized OFDM technology as a modulation method, for example, digital audio broadcasting (DAB), digital video broadcasting (DVB), as well as highperformance local area network-type 2 (HIPERLAN/2) (Siddiq, 2015; Yang et al., 2011; Chen and Kang, 2014). In addition, OFDM system also adopted by worldwide interoperability for microwave access (WiMAX) standard IEEE.802.16e/m (Alhasson and Matin, 2011), wireless local area network (WLAN) standard IEEE.802.11a/b/g/n (Jawhar et al., 2016a), and wireless personal area network (WPAN) standard IEEE.802.15.3/3a/3b/3c (Raja and Gangatharan, 2016). Both LTE and WiMAX standard of the $4 \mathrm{G}$ wireless communication system used OFDM system as a modulation technique. Moreover, OFDM is the key empowering technology for expanded version LTE-A and WiMAX 2 for the 4G system (Lee et al., 2014; Lin et al., 2013).

Although OFDM system has numerous advantages, the high PAPR of the transmission signal is considered as the main drawback in OFDM system. 
The high PAPR drives some devices such as a high power amplifier (HPA) to work in the non-linear part and leads the system to suffer from out of band emission, and in-band distortion. Hence, spectral efficiency and the bit error rate (BER) are degraded. The high PAPR is classified as the main obstacle to the OFDM system in real applications. Thus numerous algorithms have been suggested to beat the high PAPR, for example, selective-mapping (SLM) (Taher et al., 2013), partial transmit sequences (PTS) (Müller and Huber, 1997a), tone injection (Han and Lee, 2005), coding technique (Jiang and $\mathrm{Wu}, 2008$ ), clipping and filtering (Taher et al., 2014), and active constellation extension (ACE) (Krongold and Jones, 2003).

The PTS has been considered as one of the powerful PAPR decrease schemes compared to other mitigation strategies, but its computational complexity is relatively high. The principle thought of the PTS strategy is separating the block data into multiple subsets and then rotated by weighting factors to produce a compilation of the candidate signals. The signal that accomplishes minimal PAPR is selected for sending. Therefore, the PTS procedure relies on the type of the segmentation scheme used and the phase rotation vectors.

In the literature, many strategies are proposed to enhance the PAPR mitigation execution or/and diminish the computational complexity of the PTS algorithm. Among them, hybrid methods are introduced to combine a couple of conventional schemes together. Hence, the different features of the ordinary schemes can be merged in new approaches. Jawhar et al. (2016a) combined two types of the segmentation schemes to improve the PAPR diminishment performance. Similarly, Ibraheem et al. (2014) joined two ordinary schemes to reduce the PAPR greater than the conventional schemes. However, Singh and Singh (2016) merged two techniques, SLM and PTS to enhance the PAPR restraining execution. In the same manner, Pachori and Mishra (2016) integrated PTS and ACE in parallel to diminish the PAPR value accordingly.

In this paper, new PTS partitioning methods are introduced to enhance the PAPR alleviation interpretation in OFDM frameworks. The new algorithms depend on rearrangement of the interleaving segmentation scheme and the adjacent segmentation scheme to create new schemes, where the new approaches being less subcarriers correlation than conventional schemes. Furthermore, the new algorithms maintain the computational complexity at the same level of the Ad-PTS and IL-PTS.

The organization of this paper is: Section 2 introduced the OFDM and PAPR principles. The conventional partial transmit sequence is explained in Section 3. Section 4 discussed the C-PTS partitioning schemes. The proposed methods are illustrated in Section 5. Section 6 analyzed the simulation results. Lastly, the conclusion paragraph is written in Section 7.

\section{OFDM and PAPR}

In OFDM procedure, the data sequence $X$ is modulated by $N$ subcarriers to produce $X_{k}$ modulated data symbol, where the subscript $k=\{0,1,2,3, \ldots, N$ $1\}$. Then, the discrete OFDM signal $x(n)$ is obtained by applying inverse-fast-Fourier-transform (IFFT) on the $X_{k}$ symbol, with the consideration; that the subcarriers being orthogonal to each other. Therefore, the OFDM signal $x(n)$ can be written as Eq. 1 (Kim, 2016):

$x(n)=\frac{1}{\sqrt{N}} \sum_{k=0}^{N-1} X_{k} e^{j 2 \pi k n / N}, 0 \leq n \leq N-1$

where, $j=\sqrt{-1}$.

The PAPR of the OFDM signal is characterized as the proportion of the peak power of the OFDM signal divided by the mean power in the time-domain. Consequently, the PAPR in decibels $[\mathrm{dB}]$ is determined as Eq. 2 (Mhatre and Khot, 2015):

$P A P R=\frac{\max |x(n)|^{2}}{E\left\{|x(n)|^{2}\right\}}$

where, $E\{$.$\} denotes to the mean power of the signal.$ The reason behind the high PAPR value is fluctuation the signals in the time-domain, because of multiple sinusoidal functions are added together after applying IFFT on the input data. Hence, the peak power of the signal subcarriers might be greater than the mean power when the phases of these subcarriers are same (Vidya et al., 2015).

The complementary-cumulative-distributionfunction (CCDF) is utilized to quantify the probability of the PAPR surpassing a specific threshold value, which can be characterized as Eq. 3:

$P_{r}\left(P A P R\left(x(n)>P A P R_{0}\right)=1-\left(1-e^{-P A P R_{0}}\right)^{N}\right.$

where, $P_{r}$ is the probability and, PAPR0 indicates the threshold value.

\section{Conventional partial transmit sequence}

The traditional PTS (C-PTS) is a reduction method, and it is suggested to upgrade the PAPR execution in the OFDM framework (Müller and Huber, 1997b). The block diagram of the C-PTS is demonstrated in Fig. 1, in which the input modulated sequence $X$ is partitioned into $V$ non-overlapping subblocks, and then the $N$-points IFFT is performed to transform the data from the frequency-domain into the time-domain. Afterward, the transformed subblocks are rotated by weighting factors to produce a compilation of the candidate signals. Subsequently, The PAPR of each candidate signal is calculated, and signal that accomplishes minimal PAPR is chosen for sending. The optimum OFDM signal can be composed as Eqs. 4-6:

$X_{k}=\sum_{v=1}^{V} b_{v} X_{v}$

$x=\operatorname{IFFT}\left(X_{k}\right)=\operatorname{IFFT}\left\{\sum_{v=1}^{V} b_{v} X_{v}\right\}$

$x=\sum_{v=1}^{V} b_{v} \operatorname{IFFT}\left(X_{v}\right)=\sum_{v=1}^{V} b_{v} x_{v}$ 
where, $b_{v}$ are the phase rotation factors, and the subscript $v=\{1,2,3, \ldots, V\}$. The optimum phase rotation factor that realized less PAPR value can be written as Eq. 7:

$$
\left\{b_{1}, b_{2}, \ldots, b_{V}\right\} \arg \min _{\left\{b_{1}, b_{2}, \ldots, b_{V}\right\}}\left(\max \left|\sum_{v=1}^{V} b_{v} x_{v}\right|\right)
$$

where, arg $\min ($.$) is the decision condition to$ determine the smallest value.
The scope of the different rotation factors $(W)$ is usually limited by $\{\mp 1\}$ or $\{\mp 1, \mp j\}$ in order to alleviate the computational complexity for finding the optimum phase factor. Besides, the transmitter should send $\log _{2} W^{V-1}$ bits to the receiver as side information (SI), whereas SI is a substantial factor to recoup the original sequence at the receiver $(\mathrm{Wu}$ et al., 2010).

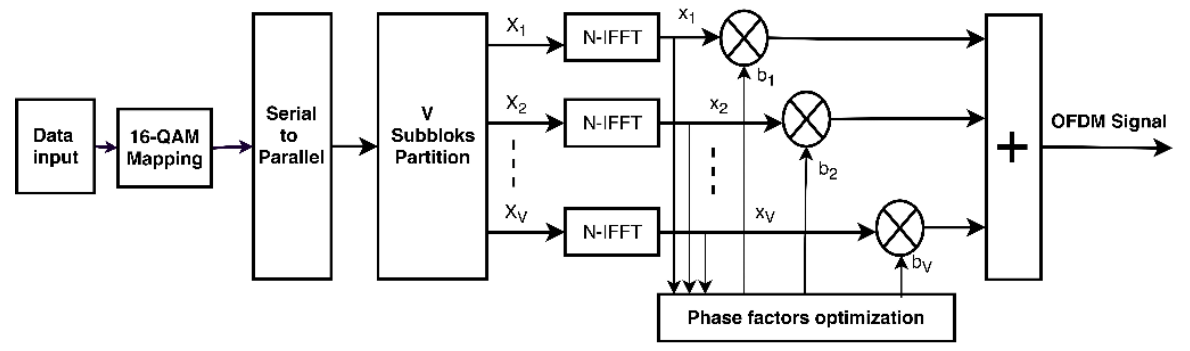

Fig. 1: C-PTS block diagram

\section{C-PTS partitioning schemes}

As mentioned, PTS technique depended on the type of the segmentation scheme and the phase rotation factors to diminish the high PAPR pattern. In C-PTS procedure, there are three kinds of the partitioning schemes involving interleaving segmentation (IL), adjacent segmentation (Ad) and, pseudo-random segmentation (PR), as presented in Fig. 2 (Kang et al., 1999). The IL partitioning scheme assigns the subcarriers with distance interval of $V$ within the subblocks. The Ad partitioning scheme allots $N / V$ sequential subcarriers inside the subblocks, successively. However, the PR partitioning scheme appropriates the subcarriers in each subblock randomly (Jawhar et al., 2016b). Moreover, the three kinds of segmentation schemes should follow two conditions: the first one is $N / V$ subcarriers must assign in each subblock, and the rest locations are set to zero, while the another condition is that each subcarrier must appear only one time within the subblock (Ibraheem et al., 2014).

The PAPR mitigation performance differs from segmentation scheme to another, depending on the subcarriers correlation within the subblocks (Xia et al., 2007). PR-PTS method has been considered the best scheme in terms of the PAPR reduction rendering, because it has the lowest correlation among its subcarriers within the subblocks. In addition, Ad-PTS is the second best scheme that can alleviate the PAPR value, due to its subcarriers correlation is stronger than that of the PR-PTS scheme. However, IL-PTS scheme counts the worse PAPR diminishment interpretation among the segmentation schemes; this can attribute of the strong correlation among its subcarriers (Lu et al., 2006).

On the other hand, the computational complexity of the PR-PTS and Ad-PTS algorithms have been recorded higher than that of the IL-PTS algorithm; this is because of the IL-PTS used Cooly-Tukey IFFT algorithm, and the periodic transition structure of the subcarriers inside each subblock, this helps to reduce the multiplications and additions IFFT operations (Hong et al., 2013).

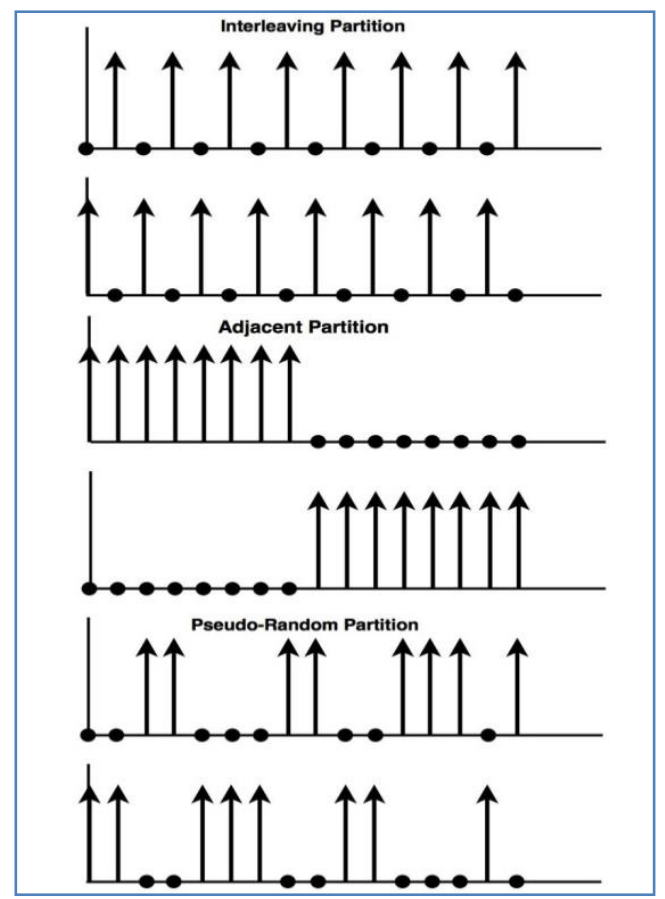

Fig. 2: Ordinary segmentation schemes

Therefore, the computational complexity of the three kinds of the partitioning schemes for the PTS technique can be written as Eqs.8-11 (Kang et al., 1999):

- The computational complexity of the PR-PTS and Ad-PTS

$C_{\text {add }}=V\left(N \log _{2} N\right)$

$C_{\text {mult }}=V\left(\frac{N}{2} \log _{2} N\right)$

- The computational complexity of the IL-PTS 
$C_{\text {add }}=V\left(\frac{N}{V} \log _{2} \frac{N}{V}\right)$

$C_{\text {mult }}=V\left(\frac{N}{2 V} \log _{2} \frac{N}{V}+N\right)$

\section{Proposed schemes}

In this section, two new PTS segmentation schemes are proposed to mitigate the high PAPR pattern in the following subsequent subsections:

\subsection{Adjacent shifting PTS scheme}

In Ad-PTS scheme, $N / V$ consecutive subcarriers are assigned inside each subblock, successively. Moreover, the PAPR performance of the Ad-PTS has been considered better than IL-PTS, and it is lower than PR-PTS depending on the correlation between the subcarriers. Further, the computational complexity of the Ad-PTS is similar to that of the PRPTS, and it records complexity higher than IL-PTS. Adjacent shifting PTS scheme (Ad-Sh-PTS) is proposed to enhance its PAPR mitigation execution greater than Ad-PTS scheme without additional computational complexity on the system.

Fig. 3 illustrates the Ad-Sh-PTS method, in which the input data sequence is divided into $S_{v}$ groups, where subscript $v=[1,2,3, \ldots, V]$, where each group contains $N / V$ subcarriers. After that, the input data sequence is segmented into $V$ subblocks similar to Ad scheme, where each row of the Ad matrix contains only one $S_{v}$ group, and the other positions are set to zero. Afterward, in every row of the $\mathrm{Ad}$ matrix, $\mathrm{S}_{v}$ group is divided into two equally parts $\mathrm{d}_{1}$ and $d_{2}$, thus the first part $d_{1}$ is fixed in its position and the second part $\mathrm{d}_{2}$ is shifted by $V-1$. This fashion continues until the last row of the Ad matrix. Finally, the newly generated matrix represents the Ad-ShPTS matrix, in which each row regards the subblock of the Ad-Sh-PTS scheme, as demonstrated in Fig. 3. The C-PTS procedure is applied to the Ad-Sh-PTS scheme, and then the optimum OFDM signal is elected for transmission.

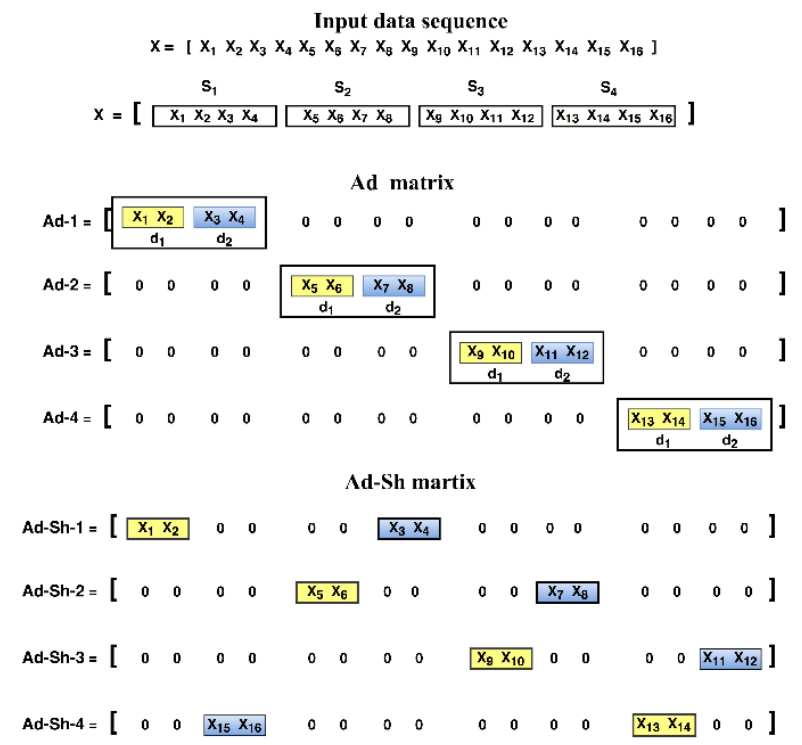

Fig. 3: Ad-Sh-PTS scheme when $V=4$ and $N=16$
Since Ad-Sh-PTS method can reduce the correlation among the subcarriers inside the subblocks, the candidate signals that generated in the time-domain after applying IFFT operation will be more independent (Xia et al., 2007). Hence the PAPR mitigation interpretation will be enhanced accordingly. Furthermore, the computational complexity of the Ad-Sh-PTS method achieves as similar as that of Ad-PTS method. Consequently, the Ad-Sh-PTS method is superior to Ad-PTS method for minimizing the PAPR value.

\subsection{Rows exchange of interleaving scheme}

In IL-PTS scheme, the subcarriers are assigned with distance interval of $V$ inside the subblocks. The PAPR performance of the IL-PTS has been regarded the worst among traditional segmentation schemes because of the strong correlation between its subcarriers. However, the computational complexity of the IL-PTS is considered lower than other segmentation schemes (Lu et al., 2006). Rows exchange of interleaving PTS scheme (IL-Ex-PTS) is introduced to enhance the PAPR alleviation rendering not only better than IL-PTS scheme but also superior to Ad-PTS scheme. However, the computational complexity of the IL-Ex-PTS fulfills higher calculations than IL-PTS scheme and similarly to that of the Ad-PTS algorithm.

The IL-Ex-PTS procedure is clarified in Fig. 4. The input sequence is segmented into $V$ subblocks by using IL scheme. Next, each row of the IL matrix is subdivided into two equal parts, the first part contains only the odd GPF groups, where the subscript $P F=\{1,3, \ldots,(1-2 V)\}$, while the second part contains only the even GPS groups, where the subscript $P S=\{2,4, \ldots, 2 \mathrm{~V}\}$. After that, in the first part, group $G_{1}$ is exchanged with group $G_{3}$, while $G_{2 V}$ group is exchanged with $\mathrm{G}_{2(\mathrm{~V}-1)}$ group in the second part of the IL matrix. Lastly, the new matrix of the ILEx-PTS scheme is obtained, as clarified in Fig. 4. The IL-Ex-PTS algorithm is implemented to the PTS procedure. Thus the OFDM signal that accomplishes minimal PAPR value is elected for sending.

The PAPR diminishment interpretation of the ILEx-PTS outperforms to both IL-PTS method and AdPTS method, this due to that the IL-Ex-PTS method has less correlation among its subcarriers than the other two. On the other hand, the computational complexity of the IL-Ex-PTS is similar to that of AdPTS method, while it is higher than IL-PTS approach, because of IL-Ex-PTS scheme broke the periodic pattern of the IL scheme. Hence the IL-Ex-PTS needs to perform all the stages of the IFFT operation.

\section{Results and discussion}

This section presents the PAPR reduction execution of the Ad-Sh-PTS and IL-Ex-PTS compared with Ad-PTS, IL-PTS, and the original OFDM signal. The PAPR interpretation is analyzed with two scenarios; the first one is performed when $V$ and $W$ are equal to 4 , while the second scenario is 
conducted when $V$ and $W$ are equal to 8 and 2 . The other parameters of the simulation are chosen as follows: the number of subcarriers $N=128$ and 256, and the CCDF is utilized to evaluate the PAPR distribution. Furthermore, 16-QAM is adopted as constellation mapping for the input data sequence; meanwhile, M-PSK (phase shifting keying) technique can be also utilized to modulate the input data sequence.

Input data sequence

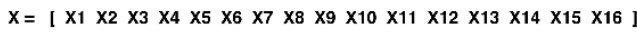

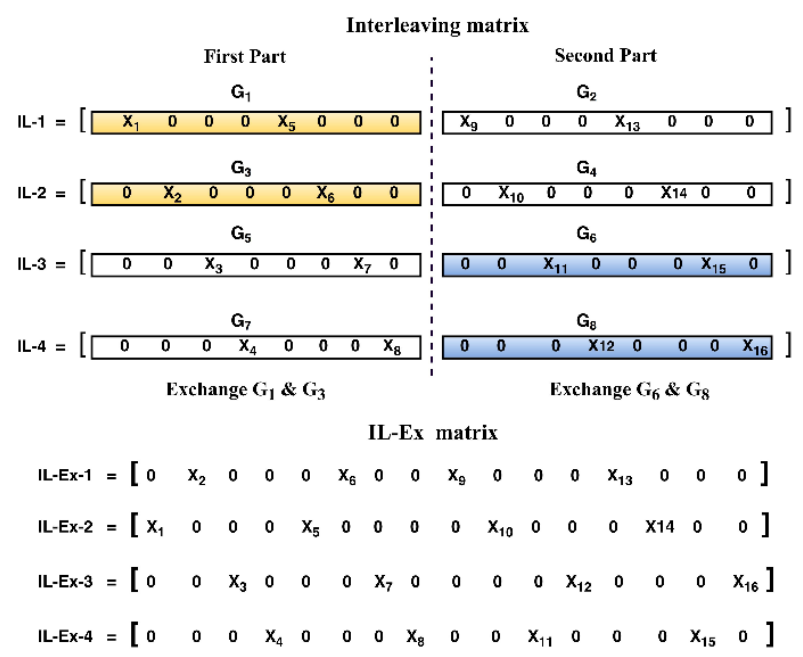

Fig. 4: IL-Ex-PTS scheme when $V=4$ and $N=16$

\subsection{Scenario A}

In this scenario, the simulation is conducted when the value of $V$ and $W$ are equal to 4 .

Fig. 5 illustrates the PAPR execution for the three types of the ordinary segmentation schemes and the original OFDM signal (without reduction). When the subcarriers $N=128$, the PAPR of the PR-PTS was $7.13 \mathrm{~dB}$, Ad-PTS was $7.85 \mathrm{~dB}$, IL-PTS was $8.45 \mathrm{~dB}$, and the original OFDM signal was 10.6dB. Likewise, Fig. 6 presented the PAPR performance when $N=256$. The PAPR of the PR-PTS was $7.61 \mathrm{~dB}$, Ad-PTS was $8.26 \mathrm{~dB}$, IL-PTS was $8.8 \mathrm{~dB}$, and the original OFDM signal was $11.05 \mathrm{~dB}$. Therefore, the PR-PTS scheme achieved better PAPR reduction rendering than both Ad-PTS and IL-PTS.

In Fig. 7 and Fig. 8, the PAPR of the Ad-Sh-PTS compared with Ad-PTS, IL-PTS, and the original OFDM signal, when $N=128$ and 256. The Ad-Sh-PTS achieved PAPR reduction to about $3.05 \mathrm{~dB}$ from the original signal, while Ad-PTS and IL-PTS reduced the PAPR value by $2.5 \mathrm{~dB}$ and $2.11 \mathrm{~dB}$ from the original signal, as shown in Fig. 7. Also, Fig. 8 indicated the Ad-Sh-PTS ability to reduce the PAPR value when $N=$ 256 , in which the reduction in PAPR of the Ad-ShPTS was $3.13 \mathrm{~dB}$ from the original signal, while AdPTS and IL-PTS were $2.66 \mathrm{~dB}$ and $2.2 \mathrm{~dB}$. Obviously, the proposed method Ad-Sh-PTS can alleviate the PAPR superior to Ad-PTS and IL-PTS with any number of subcarriers.

A comparison was presented in Fig. 9 when $N=$ 128 denoted that the PAPR execution of the IL-Ex-
PTS superior to Ad-PTS, IL-PTS, and the original OFDM signal by $0.58 \mathrm{~dB}, 0.96 \mathrm{~dB}$, and $3.08 \mathrm{~dB}$, respectively. Moreover, Fig. 10 clarified the reduction in PAPR of the IL-Ex-PTS, Ad-PTS, IL-PTS is compared with the original OFDM signal, when $N=$ 256. IL-Ex-PTS method diminished the PAPR value by $3.1 \mathrm{~dB}$, Ad-PTS by $2.75 \mathrm{~dB}$, IL-PTS by $2.24 \mathrm{~dB}$ from the original signal. Clearly, it can be seen that the ILEx-PTS scheme can minimize the PAPR value greater than Ad-PTS and IL-PTS for both fashions.

\subsection{Scenario B}

In this scenario, the simulation is conducted when the number of subblocks is 8 , and the number of the scope rotation factors equals to 2 .

Fig. 11 and Fig. 12 evaluated the three traditional segmentation schemes and the original OFDM signal when $N=128$ and 256. The PAPR interpretation of the PR-PTS surpassed Ad-PTS by $0.47 \mathrm{~dB}$, IL-PTS by $0.87 \mathrm{~dB}$, and the traditional OFDM by $3.79 \mathrm{~dB}$, as shown in Fig. 11. Furthermore, when $N=256$, the PAPR performance of the PR-PTS outperformed to Ad-PTS, IL-PTS, and the traditional OFDM by $0.3 \mathrm{~dB}$, $0.51 \mathrm{~dB}$, and $3.83 \mathrm{~dB}$, respectively. Therefore, pseudorandom is the best segmentation scheme in reducing the PAPR value for C-PTS method, and adjacent segmentation is the next best, while the interleaving partition is the worst scheme among traditional segmentation schemes.

Fig. 13 and Fig. 14 demonstrated the PAPR performance of the Ad-Sh-PTS scheme compared with Ad-PTS, IL-PTS, and the original OFDM signal, when $N=128$. As shown in Fig. 13, the Ad-Sh-PTS scheme can reduce the PAPR value about $3.66 \mathrm{~dB}$ from the original signal, while Ad-PTS achieved PAPR reduction by $3 \mathrm{~dB}$. Besides, Fig. 14 indicated that the Ad-Sh-PTS method could diminish the PAPR value maximal than Ad-PTS, IL-PTS, and the original signal by $0.21 \mathrm{~dB}, 0.43 \mathrm{~dB}$, and $3.65 \mathrm{~dB}$, respectively. Accordingly, the Ad-Sh-PTS algorithm exceeded AdPTS and IL-PTS schemes in terms of PAPR mitigation interpretation.

In the simulation results as shown in Fig. 15 and Fig. 16, the PAPR execution of the IL-Ex-PTS is evaluated in comparison with original OFDM signal and two approaches of the ordinary partitioning schemes Ad-PTS and IL-PTS. In the case of $N=128$, the PAPR of the IL-Ex-PTS was 7.01dB, Ad-PTS was 7.2 $\mathrm{dB}$, IL-PTS was $7.63 \mathrm{~dB}$, and the OFDM signal was $10.63 \mathrm{~dB}$, as demonstrated in Fig. 15. Similarly, when $N=256$, the PAPR value of the IL-Ex-PTS was $7.51 \mathrm{~dB}$, Ad-PTS was $7.66 \mathrm{~dB}$, IL-PTS was $7.88 \mathrm{~dB}$, and the traditional OFDM signal was $11 \mathrm{~dB}$, as shown in Fig.16. Consequently, the IL-Ex-PTS method can realize the reduction in PAPR value greater than AdPTS and IL-PTS methods.In summary, the simulation is conducted by two scenarios with various values of $V$ and $W$. The simulation results indicated that the two proposed methods Ad-Sh-PTS and IL-Ex-PTS could enhance the PAPR mitigation rendering preferable than both traditional schemes, Ad-PTS and IL-PTS with any number of $V$ and $W$.Table 1 and 
Table 2 recorded the PAPR values for both scenarios. It can be seen that the proposed methods Ad-Sh-PTS and IL-Ex-PTS outperform to the corresponding methods Ad-PTS and IL-PTS.

Table 1: PAPR value of the scenario $A$

\begin{tabular}{ccc}
\hline \multicolumn{3}{c}{ Scenario A; $\mathrm{V}=4, \mathrm{~W}=4$} \\
\hline PAPR [dB] \\
Method & $\mathrm{N}=128$ & $\mathrm{~N}=256$ \\
\hline Original OFDM & 10.6 & 11.05 \\
PR-PTS & 7.13 & 7.61 \\
Ad-PTS & 7.85 & 8.26 \\
IL-PTS & 8.45 & 8.8 \\
Ad-Sh-PTS & 7.36 & 7.87 \\
IL-Ex-PTS & 7.33 & 7.84 \\
\hline
\end{tabular}

Table 2: PAPR values of the scenario $B$

\begin{tabular}{ccc}
\hline \multicolumn{3}{c}{ Scenario B; $\mathrm{V}=8, \mathrm{~W}=2$} \\
\hline \multirow{2}{*}{ Method } & $\mathrm{N}=128$ & $\mathrm{~N}=256$ \\
\hline Original OFDM & 10.52 & 11.18 \\
PR-PTS & 6.73 & 7.35 \\
Ad-PTS & 7.2 & 7.65 \\
IL-PTS & 7.6 & 7.86 \\
Ad-Sh-PTS & 6.97 & 7.45 \\
IL-Ex-PTS & 7.01 & 7.51 \\
\hline
\end{tabular}

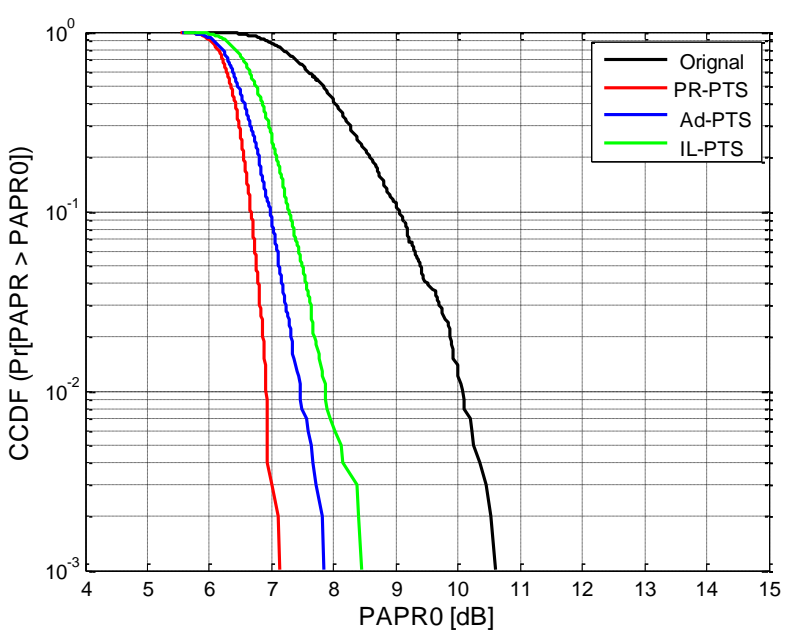

Fig. 5: Comparison ordinary segmentation schemes when $V=4, W=4$, and $N=128$

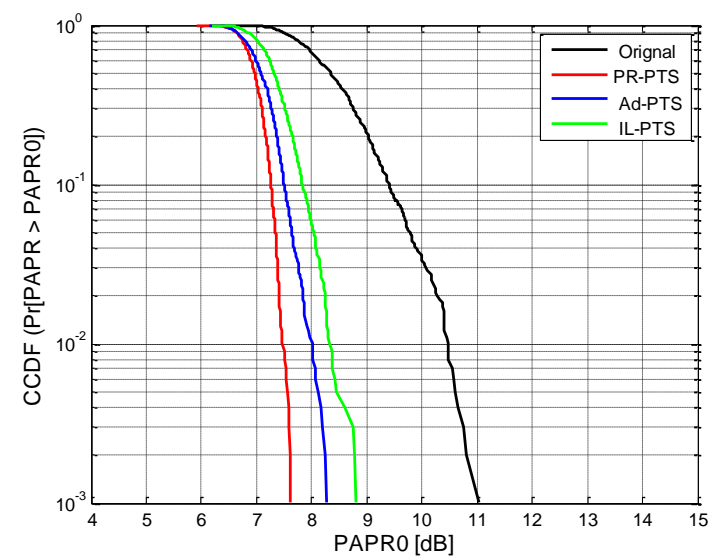

Fig. 6: Comparison ordinary segmentation schemes when $V=4, W=4$, and $N=256$

\section{Conclusion}

In this paper, two schemes of the C-PTS technique to enhance the PAPR mitigation rendering are proposed. The enhanced methods IL-Ex-PTS and IL-Ex-PTS are suggested depending on Ad-PTS and IL-PTS approaches. The proposed schemes make to decrease the correlation among the subcarriers within the partitioned subblocks in the frequencydomain.

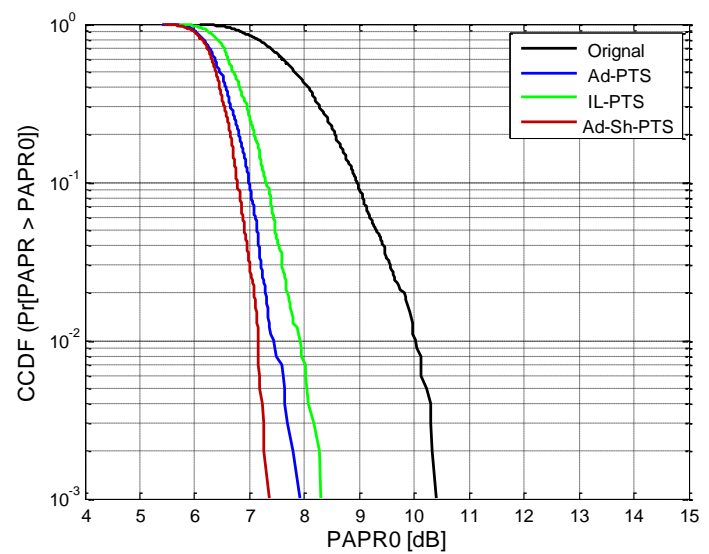

Fig. 7: Comparison Ad-Sh-PTS and ordinary schemes when $V=4, W=4$, and $N=128$

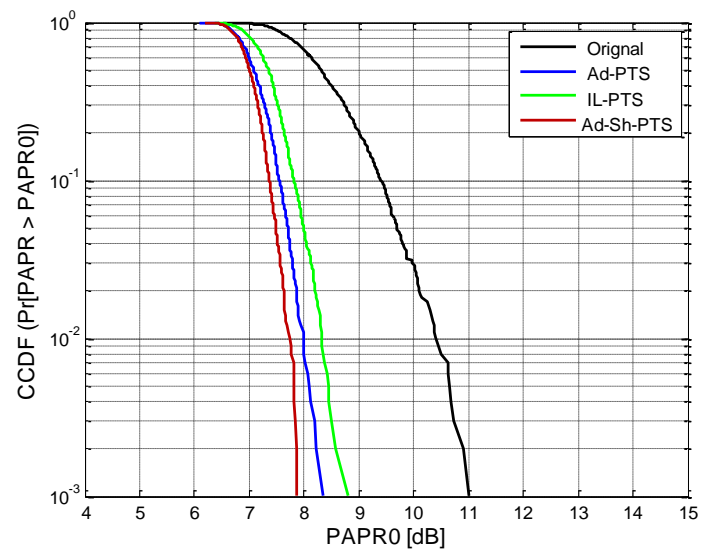

Fig. 8: Comparison Ad-Sh-PTS and ordinary schemes when $V=4, W=4$, and $N=256$

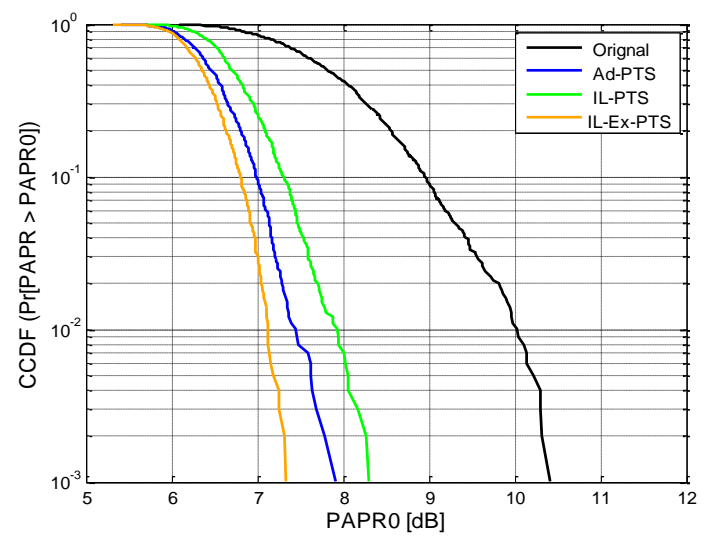

Fig. 9: Comparison IL-Ex-PTS and ordinary schemes when $V=4, W=4$, and $N=128$

Hence, the generated candidate signals in the time-domain will be more independent, and its PAPR value will be reduced accordingly. The simulation results indicated that the PAPR mitigation interpretation of the proposed approaches outperforms to Ad-PTS and IL-PTS schemes without extra complexity on the system. In future work, the 
proposed algorithm will be applied in MIMO-OPFD system.

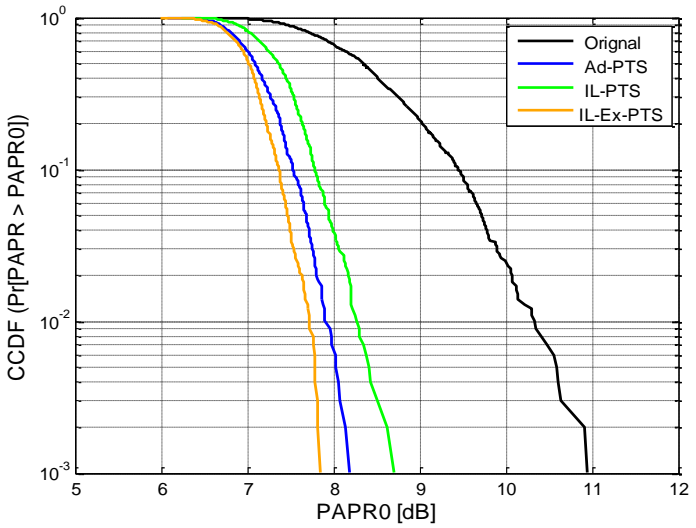

Fig. 10: Comparison IL-Ex-PTS and ordinary schemes when $V=4, W=4$, and $N=256$

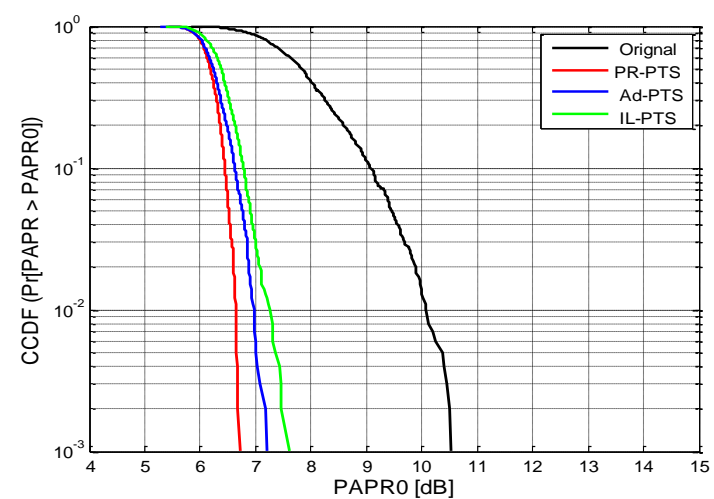

Fig. 11: Comparison ordinary segmentation schemes when $V=8, W=2$, and $N=128$

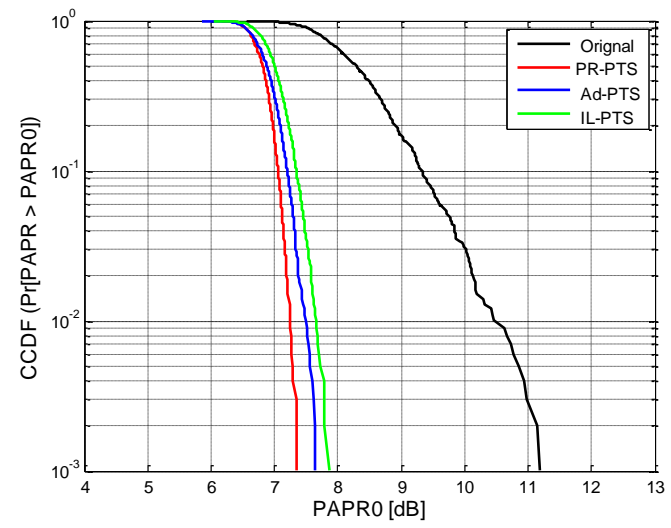

Fig. 12: Comparison ordinary segmentation schemes when $V=8, W=2$, and $N=256$

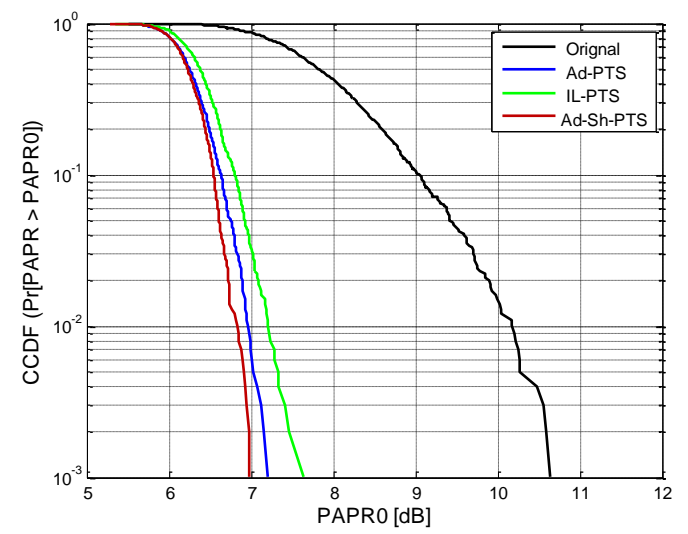

Fig. 13: Comparison Ad-Sh-PTS and ordinary schemes when $V=8, W=2$, and $N=128$

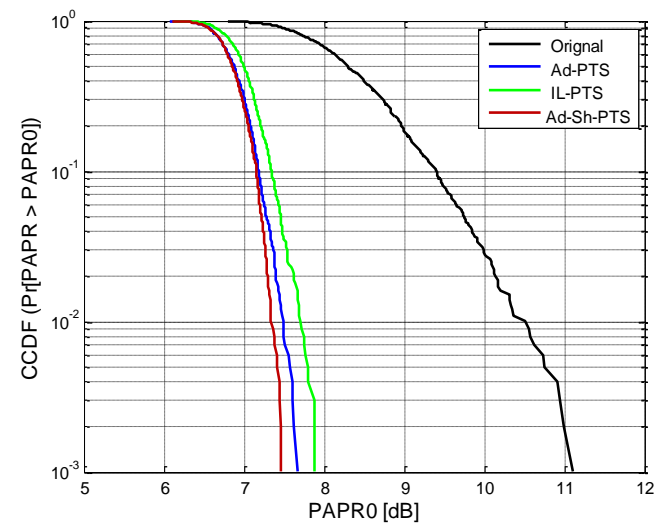

Fig. 14: Comparison Ad-Sh-PTS and ordinary schemes when $V=8, W=2$, and $N=256$

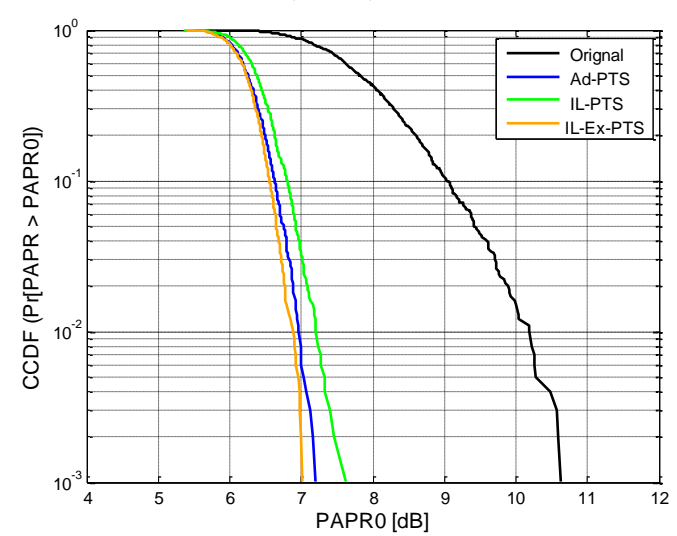

Fig. 15: Comparison IL-Ex-PTS and ordinary schemes when $V=8, W=2$, and $N=128$

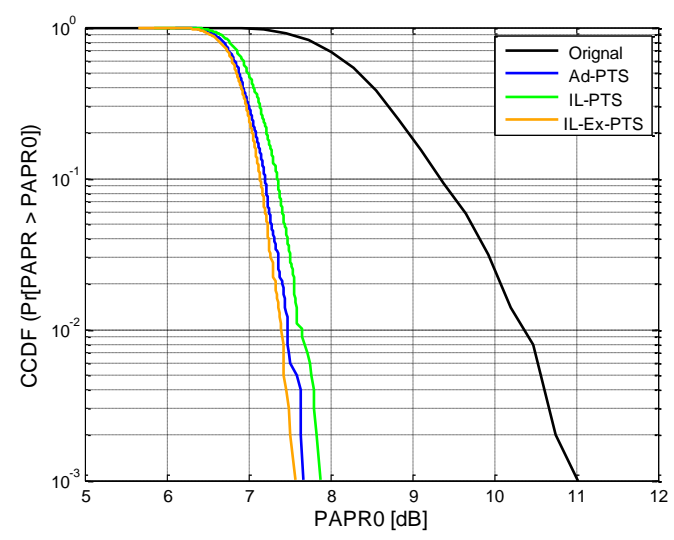

Fig. 16: Comparison IL-Ex-PTS and ordinary schemes when $V=8, W=2$, and $N=256$

\section{Acknowledgment}

The authors would like to thank to University Tun Hussein Onn Malaysia (UTHM) for sponsoring this work under Research Supporting Grant Scheme (RSGS) Vot No. U 102.

\section{References}

Alhasson B and Matin M (2011). PAPR distribution analysis of OFDM signals with partial transmit sequence. In the $14^{\text {th }}$ International Conference on Computer and Information Technology (ICCIT), IEEE: 652-656. https://doi.org/10.1109/ ICCITechn.2011.6164869

Chen Z and Kang S (2014). A three-dimensional OFDM system with PAPR reduction method for wireless sensor networks. International Journal of Distributed Sensor Networks, 2014: 
Article ID 312308, 6 pages. https://doi.org/10.1155/2014/ 312308

Han SH and Lee J (2005). An overview of peak-to-average power ratio reduction techniques for multicarrier transmission. IEEE Wireless Communications, 12(2): 56-65.

Hong C, Qin Q, and Chao T (2013). An PTS optimization algorithm for PAPR reduction of OFDM system. In the International Conference on Mechatronic Sciences, Electric Engineering and Computer (MEC), IEEE, Shenyang, China: 3775-3778. https://doi.org/10.1109/MEC.2013.6885649

Ibraheem Z, Rahman M, Yaakob S, Razalli M, Kadhim R, and Ahmed K (2014). Performance of PTS techniques with varied partition size in PAPR reduction of OFDM system. In the International Conference on Computer, Communications, and Control Technology (I4CT), IEEE, Langkawi, Malaysia: 21-25. https://doi.org/10.1109/I4CT.2014.6914138

Ibraheem Z, Rahman M, Yaakob S, Razalli M, Salman F, and Ahmed K (2014). PTS method with combined partitioning schemes for improved PAPR reduction in OFDM system. Indonesian Journal of Electrical Engineering and Computer Science, 12(11): 7845-7853.

Jawhar Y, Abdulhasan R, and Ramli K (2016a). A new hybrid subblock partition scheme of PTS technique for reduction PAPR performance in OFDM system. ARPN Journal of Engineering and Applied Sciences, 11(7): 3904-3910.

Jawhar Y, Abdulhasan R, and Ramli K (2016b). Influencing parameters in peak to average power ratio performance on orthogonal frequency-division multiplexing system. ARPN Journal of Engineering and Applied Sciences, 11(6): 43224332.

Jiang T and Wu Y (2008). An overview: peak-to-average power ratio reduction techniques for OFDM signals. IEEE Transactions on Broadcasting, 54(2): 257-268.

Kang S, Kim J, and Joo E (1999). A novel subblock partition scheme for partial transmit sequence OFDM. IEEE Transactions on Broadcasting, 45(3): 333-338.

Kim K (2016). On the shift value set of cyclic shifted sequences for PAPR reduction in OFDM systems. IEEE Transactions on Broadcasting, 62(2): 496-500.

Krongold B and Jones D (2003). PAR reduction in OFDM via active constellation extension. IEEE Transactions on Broadcasting, 49 (3): $258-268$

Lee Y, Chuah T, Loo J, and Vinel A (2014). Recent advances in radio resource management for heterogeneous LTE/LTE-A networks. IEEE Communications Surveys \& Tutorials, 16(4): 2142-2180.

Lin B, Tsai W, Wu C, Hsu P, Huang J, and Liu T (2013). The design of cloud-based 4G/LTE for mobile augmented reality with smart mobile devices. In the IEEE $7^{\text {th }}$ International Symposium on Service Oriented System Engineering (SOSE), IEEE, Redwood City, USA: 561-566. https://doi.org/ 10.1109/SOSE.2013.57

Lu G, Wu P, and Carlemalm-Logothetis C (2006). Enhanced interleaved partitioning PTS for peak-to-average power ratio reduction in OFDM systems. Electronics Letters, 42(17): 983984.

Mhatre K and Khot U (2015). Efficient selective mapping PAPR reduction technique. Procedia Computer Science, 45: 620-627.

Müller S and Huber J (1997a). A novel peak power reduction scheme for OFDM. In the $8^{\text {th }}$ IEEE International Symposium on
Personal, Indoor and Mobile Radio Communications (PIMRC'97), IEEE, Helsinki, Finland: 1090-1094. https://doi.org/10.1109/PIMRC.1997.627054

Müller S and Huber J (1997b). OFDM with reduced peak-toaverage power ratio by optimum combination of partial transmit sequences. IEEE Electronics letters, 33(5): 368-369.

Pachori K and Mishra A (2016). An efficient combinational approach for PAPR reduction in MIMO-OFDM system. Wireless Networks, 2(22): 417-425.

Praveenkumar P, Amirtharajan R, Thenmozhi K, and Rayappan J (2013). OFDM with low PAPR: A novel role of partial transmit sequence. Research Journal of Information Technology, 5(1): 35-44.

Rahmatallah Y and Mohan S (2013). Peak-to-average power ratio reduction in OFDM systems: A survey and taxonomy. IEEE Communications Surveys \& Tutorials, 15(4): 1567-1592.

Raja N and Gangatharan N (2016). A new low complexity DHT based weighted OFDM transmission for peak power reduction. Indian Journal of Science and Technology, 9(17): 14.

Siddiq A (2015). PAPR reduction in OFDM systems using peak insertion. AEU-International Journal of Electronics and Communications, 69(2): 573-578.

Singh A and Singh H (2016). Peak to average power ratio reduction in OFDM system using hybrid technique. OptikInternational Journal for Light and Electron Optics, 127(6): 3368-3371.

Taher M, Mandeep J, Ismail M, Samad S, and Islam M (2014). Reducing the power envelope fluctuation of OFDM systems using side information supported amplitude clipping approach. International Journal of Circuit Theory and Applications, 42(4): 425-435.

Taher M, Singh M, Ismail M, Samad S, and Islam M (2013). Sliding the SLM-technique to reduce the non-linear distortion in OFDM systems. Elektronika ir Elektrotechnika, 19(5): 103111.

Taspinar N and Bozkurt Y (2016). Peak-to-average power ratio reduction using backtracking search optimization algorithm in OFDM systems. Turkish Journal of Electrical Engineering \& Computer Sciences, 24(4): 2307-2316.

Vidya M, Vijayalakshmi M, and Ramalingareddy K (2015). Performance enhancement of efficient partitioning technique for PAPR reduction in MIMO-OFDM system using PTS. In the Conference on Power, Control, Communication and Computational Technologies for Sustainable Growth (PCCCTSG), IEEE, Kurnool, India 247-253. https://doi.org/10.1109/PCCCTSG.2015.7503942

Wu X, Wang J, Mao Z, and Zhang J (2010). Conjugate interleaved partitioning PTS scheme for PAPR reduction of OFDM signals. Circuits, Systems and Signal Processing, 29(3): 499-514.

Xia L, Yue X, Youxi T, and Shaoqian L (2007). A novel method to design phase factor for PTS based on pseudo-random subblock partition in OFDM system. In the IEEE $66^{\text {th }}$ Vehicular Technology Conference (VTC'07), IEEE: 1269-1273. https://doi.org/10.1109/VETECF.2007.272

Yang L, Soo K, Li S, and Siu Y (2011). PAPR reduction using low complexity PTS to construct of OFDM signals without side information. IEEE Transactions on Broadcasting, 57(2): 284290. 